Pacific Journal of Mathematic 


\title{
THE ARENS PRODUCTS AND AN IMBEDDING THEOREM
}

\author{
JULIEN HENNEFELD
}

Let $X$ be a separable Banach space, $B(X)$ be the algebra of all bounded linear operators on $X$, and $\mathscr{C}$ be the algebra of all compact linear operators. This paper centers around the general question of giving a construction of $B(X)$ as a Banach algebra starting from $\mathscr{C}$.

It is a result of Schatten and von Neumann that if $H$ is a Hilbert space, then there is an isometric imbedding of $B(H)$ onto $\mathscr{C}^{* *}$, where $\mathscr{C}^{* *}$ denotes the second dual of $\mathscr{C}$. Moreover, each of the two Arens products on $\mathscr{C}^{* *}$ coincides with the multiplication induced on $\mathscr{C}^{* *}$ by operator multiplication on $B(H)$. The proofs of these results make strong use of the Hilbert space structure.

In this paper we generalize these results to a large class of uniformly convex spaces. Moreover, we show that even when $B(X)$ is not equal to $\mathscr{C}^{* *}$ it is still possible to construct $B(X)$ as a Banach algebra starting from $\mathscr{C}$.

We now amplify the above statements. The theorem of Schatten and von Neumann is proved in [9, p. 48]. See Civin and Yood [2, p. 869] or Rickart [8, p. 289] for the result on the Arens products.

In $\S 2$ we give basic definitions and elementary results concerning Banach space bases and linear operators. In $\S 3$ we prove the existence of an isometric imbedding from $B(X)$ into $\mathscr{C}^{* *}$, under the assumption that $X$ has a shrinking, unconditionally monotone basis. Also, we show that under the same assumptions, a sufficient condition for the imbedding to be surjective is that $X$ be uniformly convex. In $\S 4$ we prove that the imbedding is surjective $\Leftrightarrow$ the two Arens products on $\mathscr{C}^{* *}$ coincide, and in that case they coincide with the multiplication on $\mathscr{C}^{* *}$ induced by operator multiplication on $B(X)$. Finally, we show that for a certain class of Banach spaces, $B(X)$ is characterized as the largest subset of $\mathscr{C}^{* *}$ in which $\mathscr{C}$ is a 2-sided ideal.

2. Preliminary definition and results.

Definition 2.1. A basis $\left(e_{j}\right)$ in a Banach space $X$ is a sequence of elements of $X$, such that for each $x \in X$, there is a unique sequence of scalars $\left(a_{j}\right)$ depending on $x$ such that $\lim _{n \rightarrow \infty}\left\|\sum_{j=1}^{n} a_{j} e_{j}-x\right\|=0$. The coefficient $a_{j}$ is called the $j^{\text {th }}$ coordinate of $x$. It is a theorem of Banach's that if you define $e_{i}^{*}$ by $e_{i}^{*}\left(e_{j}\right)=\delta_{i j}$, then $e_{i}^{*}$ is in $X^{*}$. A 
basis is called shrinking if $\left(e_{i}^{*}\right)$ is a basis for $X^{*}$. A basis is called unconditional if for each $x \in X$, the series $\sum_{j=1}^{\infty} e_{j}^{*}(x) e_{j}$ is unconditionally convergent.

Definition 2.2. If $\left(e_{j}\right)$ is a basis for $X$, let $U_{m} x=\sum_{i \leqq m} e_{i}^{*}(x) e_{i}$. Then $\left(e_{j}\right)$ is called a monotone basis if $\left\|U_{m} x\right\| \leqq\|x\|$ for all $x$ in $X$ and integers $m$.

Definition 2.3. If $\left(e_{j}\right)$ is an unconditional basis and $D$ is a subset of the positive integers, let $x^{D}=\sum_{i=1, i \in D}^{\infty} e_{i}^{*}(x) e_{i}$. It is clear that $x^{D}$ is convergent, since in a Banach space an unconditionally convergent series is also subseries convergent. Then $\left(e_{j}\right)$ is called unconditionally monotone if $\left\|x^{D}\right\| \leqq\|x\|$ for all $x$ in $X$ and subsets $D \subset \omega$.

Proposition 2.1. If $X$ is a Banach space with an unconditional basis $\left(e_{j}\right)$, then $X$ can be renormed isomorphically so that $\left(e_{j}\right)$ is an unconditionally monotone basis.

Proof. The norm $\|x\|^{\prime}=\sup \left\{\left\|x^{D}\right\|: D\right.$ is a finite subset of $\left.\omega\right\}$ is isomorphic to the original norm, and has the property that every rearrangement of $\left(e_{j}\right)$ is a monotone basis for $X[4, \mathrm{p} .73]$. Suppose that $\left(e_{j}\right)$ is not unconditionally monotone with respect to the new norm. Then there exists a subset $S \subset \omega$ such that

$$
\left\|\sum_{j=1}^{\infty} a_{j} e_{j}\right\|^{\prime}<\left\|\sum_{j \in S}^{\infty} \alpha_{j} e_{j}\right\|^{\prime} \text {. }
$$

Hence, for $n$ large enough

$$
\left\|\sum_{j \leqq n} a_{j} e_{j}\right\|^{\prime}<\left\|\sum_{j \leqq n, j \in S} a_{j} e_{j}\right\|^{\prime}
$$

But this contradicts the fact that if we rearrange the basis $\left(e_{j}\right)$ so. that we take first all the $j$ in $S$ and $\leqq n$, then it is a monotone basis.

Next we use a theorem of Maddaus to investigate $\mathscr{C}$, the space of compact operators and its dual.

Notation 2.1. $E_{i j}$ will denote the elementary matrix with a one in the $i j^{\text {th }}$ coordinate and zeros elsewhere.

Definition 2.4. By a matrix concentrated in the $j^{\text {th }}$ column (row), we will mean a matrix whose entries outside the $j^{\text {th }}$ column (row), are all zero.

Theorem 2.1. Let $X$ be a Banach space with a basis $\left(e_{j}\right)$. For 
each compact operator $A$, let $A_{n}$ be the operator whose matrix consists of the first $n$ rows of $A$ and zeros elsewhere. Then $A$ is the uniform limit of the $A_{n}$.

\section{Proof. This is proved in Maddaus [6].}

Proposition 2.2. Let $X$ be a Banach space with a basis $\left(e_{k}\right)$. Then for each fixed $j$, the set of matrices of $\mathscr{C}$ concentrated in the $j^{\text {th }}$ row is linearly isometric as a Banach space to $X^{*}$.

Proof. Let $R$ be the matrix of an operator in $\mathscr{C}$ concentrated in the $j^{\text {th }}$ row. Define $\alpha\left(e_{k}\right)=R_{j k}$ and extend $\alpha$ linearly to finite linear combinations of $\left(e_{k}\right)$. Let $x=\sum_{k=1}^{n} b_{k} e_{k}$. Then $\alpha(x)=\sum_{k=1}^{n} b_{k} R_{j k}$ and $R(x)=\left(\sum_{k=1}^{n} b_{k} R_{j k}\right) e_{j}$. Then since $|\alpha(x)|=\|R(x)\|$ for each such $x, \alpha$ can be extended to a functional $\alpha \in X^{*}$ and the map $R \mapsto \alpha$ is isometric. This map is surjective because given $\alpha \in X^{*}$, define the matrix $R$ concentrated in the $j^{\text {th }}$ row with $R_{j k}=\alpha\left(e_{k}\right)$.

Proposition 2.3. Let $X$ be a Banach space with an unconditionally monotone basis $\left(e_{k}\right)$. Then for each fixed $j$ the set of matrices of $\mathscr{C}$ concentrated in the $j^{\text {th }}$ column is linearly isometric as a Banach space to $X$.

Proof. Let $C_{j}$ be a matrix in $\mathscr{C}$ concentrated in the $j^{\text {th }}$ column. Consider the map $C_{j} \mapsto C_{j} e_{j}$. Clearly $\left\|C_{j} e_{j}\right\| \leqq\left\|C_{j}\right\|$. For the other inequality, consider $x=b_{j} e_{j}+\sum_{i \neq j} b_{i} e_{i}$ with $\|x\|=1$. Then by unconditional monotonicity $\left|b_{j}\right| \leqq 1$. Hence,

$$
\left\|C_{j} x\right\|=\left\|C_{j}\left(b_{j} e_{j}\right)\right\| \leqq\left\|C_{j} e_{j}\right\| \text {. }
$$

Proposition 2.4. Let $X$ be a Banach space with a shrinking basis $\left(e_{j}\right)$. Then, with each $f$ in $\mathscr{C}^{*}$ we can associate a matrix so that $f=g \Longleftrightarrow$ their matrices coincide.

Proof. First, we will show that the marices with a finite number of nonzero entries span a dense linear manifold of $\mathscr{C}$.

Given a compact operator $A$ and $\varepsilon>0$, choose $n$ so that $\left\|A-A_{n}\right\|<$ $(\varepsilon / 2)$, where $A_{n}$ is the matrix consisting of the first $n$ rows of $A$. Let $R_{j}$ be the operator $A_{n}$ followed by the canonical projection onto the 1-dimensional subspace spanned by $\left[e_{j}\right]$, for $j=1,2, \cdots, n$. The matrix for $R_{j}$ is simply the $j^{\text {th }}$ row of $A_{n}$ and all other rows zero. Using the fact that the map in Proposition 2.2. is isometric and the hypothesis that $\left(e_{k}\right)$ is a shrinking basis, it follows that each of the matrices $R_{j}$ can be approximated to within $\varepsilon / 2 n$ by deleting (i.e., re- 
placing by zeros) the tail of the $j^{\text {th }}$ row. Therefore, by the triangle inequality $A$ can be approximated to within $\varepsilon$ by a finite matrix.

For $f$ in $\mathscr{C}^{*}$ we can define the matrix $\left(f_{i j}\right)$ by $f_{i j}=f\left(E_{i j}\right)$. Then if $f$ and $g$ have the same matrices they are equal.

Proposition 2.5. Suppose $X$ is a Banach space with an unconditionally monotone basis $\left(e_{j}\right)$ and $T$ is in $B(X)$. Then the matrix obtained by deleting (i.e., replacing by zeros) any set of rows or columns from $T$ is in $B(X)$ and has norm $\leqq\|T\|$.

Proof. Fix a subset $D \subset \omega$. Define $P x=\sum_{j \in D}^{\infty} e_{j}^{*}(x) e_{j}$. Then, $\|T P(x)\| \leqq\|T\|\|P x\| \leqq\|T\|\|x\|$. Thus, $\|T P\| \leqq\|T\|$. Also note that the matrix for $T P$ is formed by deleting the $j^{\text {th }}$ column from $T$ for every $j \notin D$.

Similarly, $\|P T\| \leqq\|T\|$ and the matrix for $P T$ is formed by deleting the $j^{\text {th }}$ row from $T$ for every $j \notin D$.

Proposition 2.6. Suppose $X$ is a Banach space with an unconditionally monotone, shrinking basis $\left(e_{j}\right)$, and that $f$ is in $\mathscr{C}^{*}$. Then the matrix obtained by deleting any set of rows or columns from the associated matrix for $f$, is the matrix associated with a functional in $\mathscr{C}^{*}$ with norm $\leqq\|f\|$.

Proof. Fix a subset $D \subset \omega$. Let $d: \mathscr{C} \rightarrow \mathscr{C}$ be the linear transformation which deletes the $j^{\text {th }}$ column for each $j \notin D$. Then its adjoint $d^{*}$ has norm 1 . Note that $\left(d^{*} f\right) A=f(d A)$. Hence, the matrix for $d^{*} f$ is formed by deleting every $j^{\text {th }}$ column for $j \notin D$.

The argument for deleting rows is similar.

Proposition 2.7. Let $X$ be a Banach space with an unconditionally monotone, shrinking basis.

(1) For each fixed $j$, the set of matrices in $\mathscr{C}^{*}$ which are concentrated in the $j^{\text {th }}$ row is linearly isometric as a Banach space to $X^{* *}$.

(2) For each fixed $j$, the set of matrices in $\mathscr{C}^{*}$ which are concentrated in the $j^{\text {th }}$ column is linearly isometric to $X^{*}$.

Proof. (1) Let $f_{j} \in \mathscr{G}^{*}$ be concentrated in the $j^{\text {th }}$ row. Define $\phi\left(e_{k}^{*}\right)=f_{j k}$. Extend $\phi$ linearly to finite linear combinations of $\left(e_{k}^{*}\right)$. It follows from Proposition 2.2 that $\dot{\rho}$ can be extended to a functional in $X^{* *}$. Moreover, $\|\dot{\rho}\|=\left\|f_{j}\right\|$ since $f_{j}$ approaches its norm on compact operators of norm one, concentrated in the $j^{\text {th }}$ row. The map $f_{j} \mapsto \phi$ is surjective because given $\phi \in X^{* *}$, the matrix whose $j^{\text {ih }}$ row is given by $f_{j k}=\left(e_{k}^{*}\right)$ and whose other rows are zero is in $\mathscr{C}^{*}$. 


\section{(2) The proof is similar.}

3. An imbedding theorem. We are now ready to give an isometric imbedding of $B(X)$ into $\mathscr{C}^{* *}$.

THEOREM 3.1. If $\left(e_{j}\right)$ is an uncondionally monotone, shrinking basis for the Banach space $X$, then there is a linear isometric map from $B(X) \rightarrow \mathscr{C}^{* *}$ such that each $A$ in $\mathscr{C}$ is taken onto its usual image under the evaluation map of $\mathscr{C} \rightarrow \mathscr{C}^{* *}$.

Proof. Given $T$ in $B(X)$ let $R_{j}$ be the matrix consisting of the $j^{\text {th }}$ row of $T$ with zeros elsewhere. Define $\Phi_{T}$ in $\mathscr{C}^{* *}$ by $\Phi_{T}(f)=$ $\sum_{j=1}^{\infty} f\left(R_{j}\right)$, where $f$ is in $\mathscr{C}^{*}$ and $\|f\|=1$. We must show that the series $\sum_{j=1}^{\infty} f\left(R_{j}\right)$ is convergent. By Proposition 2.5.

$$
\left|f\left(R_{j_{1}}+\cdots+R_{j_{n}}\right)\right| \leqq\|T\|
$$

for an arbitrary set of integers $\left\{j_{1}, \cdots, j_{n}\right\}$, since the left side represents $f$ applied to a compact operator formed by deleting rows from $T$. It is clear then that the series $\sum_{j=1}^{\infty} f\left(R_{j}\right)$ is unconditionally convergent.

The map $T \mapsto \Phi_{T}$ is obviously linear, since matrix addition and taking limits are linear operations.

$$
\left|\Phi_{T}(f)\right|=\left|\sum_{j=1}^{\infty} f\left(R_{j}\right)\right|=\lim _{n \rightarrow \infty}\left|f\left(\sum_{j=1}^{n} R_{j}\right)\right| \leqq\|f\|\|T\|,
$$

since $\sum_{j=1}^{n} R_{j}$ is a compact operator of norm $\leqq\|T\|$. Hence, $\Phi_{T}$ is bounded and $\left\|\Phi_{T}\right\| \leqq\|T\|$. To prove the reverse, first, we note that $\left\|\sum_{j=1}^{n} R_{j}\right\|$ approaches $\|T\|$ as $n$ approaches $\infty$. Then, given $\varepsilon>0$, take $\left\|\sum_{j=1}^{n} R_{j}\right\|>\|T\|-\varepsilon$. Since $\sum_{j=1}^{n} R_{j}$ is compact, we can find by the Hahn Banach theorem a $g$ in $\mathscr{C}^{*}$ of norm 1 , such that

$$
g\left(\sum_{j=1}^{n} R_{j}\right)>\|T\|-\varepsilon .
$$

Then let $g^{D}$ be the matrix formed by deleting the columns of $g$ past the $n^{\text {th }}$. By Proposition 2.6., $\left\|g^{D}\right\| \leqq 1$, and we have that $\Phi_{T}\left(g^{D}\right)>$ $\|T\|-\varepsilon$. Hence, $\left\|\Phi_{T}\right\| \geqq\|T\|$ and the imbedding is isometric.

Then as we noted in Proposition 2.4., the finite matrices form a dense manifold of $\mathscr{C}$. It is clear that $\Phi$ and the evaluation map agree on all finite matrices in $\mathscr{C}$ and hence on all of $\mathscr{C}$.

Proposition 3.1. Let $X$ be a Banach space with an unconditionally monotone, shrinking basis. Then $B(X)=\mathscr{C}^{* *}$ under the previous imbedding $\Leftrightarrow$ the set of finite matrices in $\mathscr{C}^{*}$ is a dense 
linear manifold. Moreover, in that case $X$ is reflexive.

Proof. If the set of finite matrices is not dense in $\mathscr{C}^{*}$, then there exists a nonzero $F$ in $\mathscr{C}^{* *}$, which is 0 on all finite matrices. However no $\Phi_{T}$ for nonzero $T$ in $B(X)$ can have this property, since if $T$ has the entry $T_{i j} \neq 0$, then $\Phi_{T}\left(f_{i j}\right)=T_{i j}$ where $f_{i j}$ is an elementary matrix in $\mathscr{C}^{*}$.

Assume the finite matrices are dense in $\mathscr{C}^{*}$. Let $\pi$ be an arbitrary functional in $X^{* *}$. Then by Proposition 2.7., $\pi$ can be identified with an $f \in \mathscr{C}^{*}$ which is concentrated in the $j^{\text {th }}$ row. Since the finite matrices are dense in $\mathscr{C}^{*}, \sum_{k=1}^{\infty} f_{j k} \widehat{e}_{k}$ converges in norm to $\pi$ and hence $X$ is reflexive.

Given $F \in \mathscr{C}^{* *}$, define the matrix $\left(F_{i j}\right)$ by $F_{i j}=F\left(f_{i j}\right) . F$ is determined by this associated matrix. By reflexivity and Proposition 2.7., it follows that each column of $F$ represents an element of $X$ with respect to $\left(e_{j}\right)$. Then let $T_{n}$ be the matrix consisting of the first $n$ columns of $F$. It is the matrix of a compact operator. Furthermore $\Phi_{T_{n}}(f)=F\left(f^{D}\right)$ for each $f \in \mathscr{C}^{*}$, where $f^{D}$ is the matrix formed from $f$ by deleting all the columns past $n^{\text {th }}$. Hence, $\left\|T_{n}\right\|=$ $\left\|\Phi_{T_{n}}\right\| \leqq\|F\|$. Define the operator $T$ by $T\left(\sum_{j=1}^{n} \alpha_{j} e_{j}\right)=T_{n}\left(\sum_{j=1}^{n} a_{j} e_{j}\right)$. $T$ is well defined on the set of all finite linear combinations of the $\left(e_{j}\right)$, and has norm $\leqq\|F\|$. Hence, it can be extended uniquely to a bounded operator on all of $X$. It is clear that $F=\Phi_{r}$, since $F$ and $\Phi_{T}$ agree on all finite matrices in $\mathscr{C}^{*}$.

The next proposition puts Proposition 3.2. into a more workable form for applications.

Proposition 3.2. Let $X$ be a Banach space with an unconditionally monotone shrinking basis $\left(e_{j}\right)$. Then, $B(X)=\mathscr{C}^{* *} \Leftrightarrow$ for each $f$ in $\mathscr{C}^{*},\left\|f^{N}\right\| \rightarrow 0$, where $f^{N}$ is the matrix formed from $f$ by deleting the first $N$ rows and $N$ columns.

Proof. We will show that the condition on the right is satisfied $\Leftrightarrow$ the set of finite matrices in $\mathscr{C}^{*}$ span a dense manifold.

Suppose that the finite matrices are norm dense in $\mathscr{C}^{*}$. Given $\varepsilon>0$ and $f \in \mathscr{C}^{*}$ there exists a finite $g$ such that $\|f-g\|<\varepsilon$. Then since $g$ is finite we can pick $N$ large enough so that $f^{N}=(f-g)^{N}$. By Proposition 2.6. $\left\|(f-g)^{N}\right\| \leqq\|f-g\|<\varepsilon$.

Conversely, suppose $\left\|f^{N}\right\| \rightarrow 0$. Given $\varepsilon>0$ choose $N$ large enough: $\left\|f^{N}\right\|=\left\|f-\left(f-f^{v}\right)\right\|<\varepsilon / 2$. The matrix for $f-f^{N}$ is not finite, but can be approximated to within $\varepsilon / 2$ by a finite matrix.

The next proposition shows that if $B(X) \neq 6^{* *}$, then the Banach space $X$ behaves very much like $\left(c_{0}\right)$, the space of sequences which 
converge to 0.

Proposition 3.3. Let $X$ be a Banach space with an unconditionally monotone shrinking basis $\left(e_{j}\right)$. If $B(X) \neq \mathscr{C}^{* *}$, then for every $\varepsilon>0$, and integer $n$, we can find an $x$ of norm 1 , such that $x=x_{1}+\cdots+x_{n}$, where each $x_{i}$ is a finite linear combination of distinct sets of basis vectors and $\left\|x_{i}\right\| \geqq 1-\varepsilon$.

Proof. By the previous proposition there exists an $f$ in $\mathscr{C}^{*}$ such that $\left\|f^{N}\right\|$ does not approach 0 . The $f^{N}$ decrease in norm, since $f^{N+1}$ is formed by deleting a row and a column from $f^{N}$. We can assume without loss of generality that $\left\|f^{N}\right\| \rightarrow 1$ and never achieve it as $N \rightarrow \infty$. Then, given $\lambda>0$, there exists an integer $N_{1}:\left\|f^{N_{1}}\right\|<1+\lambda$. Since the finite operators are dense in the compact operators there exists an integer $N_{1}^{\prime}>N_{1}$, and a finite operator $T_{1}$ of norm 1: $T_{1}$ is concentrated on the manifold $X_{1}$ spanned by $\left[e_{N_{1}}, \cdots, e_{N_{1}^{\prime}}\right]$ and $f^{N_{1}}\left(T_{1}\right)>1$. Let $N_{2}=N_{1}^{\prime}+1$. For $f^{N_{2}}$ there exists a finite operator $T_{2}$ of norm 1 , concentrated on the manifold $X_{2}=\left[e_{N_{2}}, \cdots, e_{N_{2}^{\prime}}\right]: f^{N_{2}}\left(T_{2}\right)>1$. Repeating this process $n$ times, we can construct $T_{1}, \cdots, T_{n}$ such that $f^{N_{k}}\left(T_{k}\right)>1$, and the $T_{k}$ are concentrated on disjoint basic blocks of $X$. Hence

$$
\begin{aligned}
n & <f^{N_{1}}\left(T_{1}\right)+\cdots+f^{N_{n}}\left(T_{n}\right)=f^{N_{1}}\left(T_{1}+\cdots+T_{n}\right) \\
& \leqq\left\|f^{N_{1}}\right\|\left\|T_{1}+\cdots+T_{n}\right\|,
\end{aligned}
$$

and $n / 1+\lambda<\left\|T_{1}+\cdots+T_{n}\right\|$. This means that there exists an $x$ of norm 1 , where $x=x_{1}+\cdots+x_{n}$, each $x_{i}$ is in $X_{i}$, and such that

$$
\frac{n}{1+\lambda}<\left\|\left(T_{1}+\cdots+T_{n}\right) x\right\| \leqq\left\|T_{1} x_{1}\right\|+\cdots+\left\|T_{n} x_{n}\right\| .
$$

However, $\lambda>0$ was arbitrary. By picking $\lambda>0$ small enough, we can find $T_{1}, \cdots, T_{n}$ : the sum $\left\|T_{1} x_{1}\right\|+\cdots+\left\|T_{n} x_{n}\right\|$ is as close to $n$ as we wish. By unconditional monotonicity, each $\left\|x_{i}\right\| \leqq 1$. Thus, $\left\|T_{i} x_{i}\right\| \leqq 1$. Hence, each $\left\|T_{i} x_{i}\right\|$ and $\left\|x_{i}\right\|$ will be close to 1 .

LEMma 3.1. A uniformly convex Banach space is reflexive.

Proof. See Wilansky [10, p. 109].

Lemma 3.2. If $X$ is a reflexive Banach space with a basis, then the basis is shrinking.

Proof. See [10, p. 213].

THEOREM 3.2. If $X$ has an unconditionally monotone basis $\left(e_{j}\right)$ 
and $X$ is isomorphic to a uniformly convex Banach space $Z$, then $B(X)=\mathscr{C}^{* *}$.

Proof. For each $x$ in $X$ call its norm $\|x\|$, and for its image in $Z$ call its norm $|x|$. Uniform convexity means that for every $\varepsilon>0$, there exists a $\delta(\varepsilon)>0$ such that if $x, x^{\prime}$ are in the unit ball of $Z$, and $\left|x-x^{\prime}\right|>\varepsilon$, then $\left|x+x^{\prime}\right| / 2 \leqq 1-\delta(\varepsilon)$. Clearly, if we renorm $Z$ by multiplying the old norm by some constant, the renormed $Z$ will still be uniformly convex. Hence, we may assume without loss of generality that there exists a constant $M:\|x\| \leqq|x| \leqq M\|x\|$. Let $t=\delta(1 / 2 M)$. Choose $r$ large enough so that, $(1 / 1-t)^{r}(1 / 2 M)>1$. Suppose $B(X) \neq \mathscr{C}^{* *}$. By Proposition 3.3. there exists an $x$ of norm 1 , such that $x=x_{1}+\cdots+x_{2^{r}}$, where each $\left\|x_{i}\right\| \geqq 1 / 2$ and where each $x_{i}$ is a linear combination of distinct $\left(e_{j}\right)$. We want to construct an element $v:\|v\|>1$ and $|v| \leqq 1$. This will contradict the fact that $\|v\| \leqq|v|$.

Consider the following system of elements like the seeding chart of a tennis tournament. In the first round put the elements $w_{1}, \cdots, w_{2}$ where $w_{k}=\left(x_{1}+\cdots+x_{k}\right) / M$ and $x_{i}$ as above. Then we construct the second round consisting of $2^{r-1}$ elements by letting the $n^{\text {th }}$ element of the second round be $u_{n}=\left(w_{2 n-1}+w_{2 n}\right) / 2(1-t)$. To form the $n^{\text {th }}$ element $y_{n}$ of the third round, let

$$
y_{n}=\frac{1}{2(1-t)}\left(u_{2 n-1}+u_{2 n}\right) \text {. }
$$

The elements for the other rounds are formed in the same manner.

We claim that every element in this system lies in the unit ball of $Z$. For the first round, each $w_{k}$ is in the unit ball of $Z$, because $\left\|w_{k}\right\| \leqq 1 / M$ by unconditional monotonicity. We can assume that two paired elements $u$ and $u^{\prime}$ from the $n^{\text {th }}$ round are in the unit ball of $Z$. Note that there exists an $x_{k}: u^{\prime}=\left(1 / M(1-t)^{n-1}\right) x_{k}+$ other terms not involving $x_{k}$, whereas $u$ does not involve any of the $\left(e_{i}\right)$ used in expressing $\left(x_{k}\right)$. By unconditional monotonicity

$$
\left\|u-u^{\prime}\right\| \geqq \frac{1}{M}\left\|x_{k}\right\| \geqq \frac{1}{2 M} .
$$

Hence,

$$
\left|u-u^{\prime}\right| \geqq \frac{1}{2 M} \text { and }\left|\frac{1}{2(1-t)}\left(u+u^{\prime}\right)\right| \leqq 1 .
$$

Thus an arbitrary element of the $(n+1)^{s t}$ round is in the unit ball of $Z$. Let $v$ be the element in the $r^{\text {th }}$ round. Then, $v=\left\{1 /(1-t)^{r} M\right\} x_{1}+$ other terms not involving $x_{1}$. Hence $\|v\|>1$. This is impossible 
since $|v| \leqq 1$.

CoRollary 3.1. If $X$ is isomorphic to a uniformly convex space and has an unconditional basis, then $B(X)$ is isomorphic to $\mathscr{C}^{* *}$.

Proof. Renorm $X$ so that the basis is unconditionally monotone.

EXAMPLE 3.1. The canonical basis for $l^{p}$ for $1<p<\infty$ is unconditionally monotone and $l^{p}$ is uniformly convex, see Clarkson [3]. $L^{p}[0,1]$ for $1<p<\infty$, has an unconditional basis and is uniformly convex. See Pelczynski [7].

4. The Arens products. The two Arens products are defined in stages according to the following rules. Let $\mathscr{A}$ be a Banach algebra. Let $A, B \in \mathscr{A} ; f \in \mathscr{A}^{*} ; F, G \varepsilon^{* *}$.

DeFinition 4.1.

$\left(f_{1}^{*} A\right) B=f(A B)$. This defines $f_{1}^{*} A$ as an element of $\mathscr{A}^{*}$. $\left(G_{1}^{*} f\right) A=G\left(f_{1}^{*} A\right)$. This defines $G_{1}^{*} f$ as an element of $\mathscr{A}^{*}$. $\left(F_{1}^{*} G\right) f=F\left(G_{1}^{*} f\right)$. This defines $F_{1}^{*} G$ as an element of $\mathscr{A}^{* *}$. We will call $F_{1}^{*} G$ the first Arens product, or the $m_{1}$ product.

DEFINITION 4.2.

$\left(A_{2}^{*} f\right) B=f(B A)$. This defines $A_{2}^{*} f$ as an element of $\mathscr{A}^{*}$. $\left(f_{2}^{*} F\right) A=F\left(A_{2}^{*} f\right)$. This defines $f_{2}^{*} F$ as an element of $\mathscr{A}^{*}$. $\left(F_{2}^{*} G\right) f=G\left(f_{2}^{*} F\right)$. This defines $F_{2}^{*} G$ as an element of $\mathscr{A}^{* *}$. $F_{2}^{*} G$ is the second Arens product or the $m_{2}$ product.

It is proved in Arens [1] that $m_{1}$ and $m_{2}$ are both Banach algebra products on $\mathscr{A}^{* *}$, which extend the original multiplication on $\mathscr{A}$ when it is imbedded in $\mathscr{A}^{* *}$.

Definition 4.3. A Banach algebra $\mathscr{A}$ is called Arens regular if the two Arens products coincide on $\mathscr{A}^{* *}$.

DEFINITION 4.4. Let $E_{\alpha}$ be a net of elements in the unit ball of $\mathscr{A}$. Then $E_{\alpha}$ is a weak identity if for every $A \in \mathscr{A}, f \in \mathscr{A}^{*}$, both $f\left(E_{\alpha} A\right) \rightarrow f(A)$ and $f\left(A E_{\alpha}\right) \rightarrow f(A)$.

Lemma 4.1. If $\mathscr{A}$ has a weak identity $E_{\alpha}$, then there exists an element $I \in \mathscr{A}^{* *}$, which is simultaneously (1) a right identity for $m_{1}$ (2) a left identity for $m_{2}$. Call such an element $I$ a simultaneous identity. 
Proof. (1) is proved in [2, p. 855]. The proof of (2) is similar. A subnet of the $\left\{E_{\alpha}\right\}$ converges to $I$ in the weak star topology.

Definition 4.5. Let $X$ be a normed space. Then, $f_{\alpha} \rightarrow f$ in the bounded weak star topology $\Leftrightarrow$ the $\left\{f_{\alpha}\right\}$ consititute a bounded set and $f_{\alpha} \rightarrow f$ in the weak star topology.

LEMma 4.2. $\mathscr{A}$ is Arens regular $\Leftrightarrow$ there is a multiplication

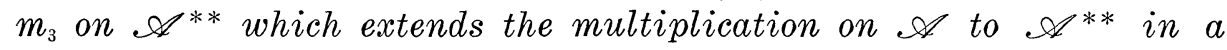
way such that (1) $F_{3}^{*} G$ is weak star bounded continuous in $F$ for each fixed $G$ and (2) $F_{3}^{*} G$ is weak star bounded continuous in $G$ for each fixed $F$.

\section{Proof. Arens [1, p. 843].}

THEOREM 4.1. If $X$ is a Banach space with an unconditionally monotone, shrinking basis $\left(e_{j}\right)$, then $B(X)=\mathscr{C}^{* *} \Leftrightarrow \mathscr{B}$ is Arens regular.

Proof. Assume $B(X)=\mathscr{C}^{* *}$. We claim that ordinary matrix multiplication satisfies (1) and (2) of the above lemma. Let $S_{\alpha}, S$, and $T$ all be in the unit ball of $B(X)$ and $S_{\alpha} \rightarrow S$ weak star. Let $f_{i j}$ be the matrix in $\mathscr{C}^{*}$ with a 1 in the $i j^{\text {th }}$ coordinate and zeros elsewhere. First, we claim that $\left(S_{\alpha} T\right) f_{i j} \rightarrow(S T) f_{i j}$. Clearly, only the $i^{\text {th }}$ rows of $S_{\alpha}$ and $S$ and the $j^{\text {th }}$ column of $T$ are relevant. By Proposition 2.3. given $\varepsilon>0$, there exists an integer $n$ such that the tail of the $j^{\text {th }}$ column of $T$ after the first $n$ terms has norm $<\varepsilon / 2$.

Since $S_{\alpha} \rightarrow S$ weak star, it is clear that $S_{\alpha}$ approaches $S$ coordinatewise. Let $\alpha$ be large enough so that each of the first $n$ entries of the $i^{\text {th }}$ row of $S$ are within $\varepsilon / 2 n$ of the corresponding entry of $S$. Then $\left|\left(S_{\alpha} T\right) f_{i j}-(S T) f_{i j}\right| \leqq \varepsilon$. Hence, $\left(S_{\alpha} T\right) f_{i j} \rightarrow(S T) f_{i j}$. Since $B(X)=$ $\mathscr{C}^{* *}$ implies that the finite matrices are norm dense in $\mathscr{C}^{*}$, it follows that for arbitrary $g \in \mathscr{C}^{*},\left(S_{\alpha} T\right) g \rightarrow(S T) g$. The argument that (2) is satisfied is similar.

Now assume $B(X) \neq \mathscr{C}^{* *}$. Then the finite matrices do not span a dense manifold of $\mathscr{C}^{*}$. Hence, there exists a nonzero $F$ in $\mathscr{C}^{* *}$ which is 0 on all finite matrices. Let $E_{n}$ be the matrix in $\mathscr{C}$ with ones down the first $n$ entires of the diagonal and zeros elsewhere. Then, $\left(E_{n}\right)$ is a weak identity since it is actually an approximate identity by the fact that finite matrices are norm dense in $\mathscr{C}$.

Let $I$ be the simultaneous identity in Lemma 4.1., and $f \in \mathscr{C}^{*}$. By Theorem 3.2. [1]

$$
\begin{aligned}
\left(F_{2}^{*} I\right) f & =\lim \left[\left(F_{2}^{*} E_{n}\right) f\right]=\lim \left[E_{n}\left(f_{2}^{*} F\right)\right] \\
& =\lim \left[\left(f_{2}^{*} F\right) E_{n}\right]=\lim \left[F\left(E_{n 2}^{*} f\right)\right] .
\end{aligned}
$$


However, $E_{n 2}^{*} f$ is the matrix in $\mathscr{C}^{*}$ which consists of the first $n$ columns of $f$, and thus can be approximated in norm by a finite matrix, since the basis is shrinking. Hence $\left(F_{2}^{*} I\right)=0$ whereas $F_{1}^{*} I=F$.

LEMmA 4.3. If there is a continuous homomorphism of the Banach algebra $\mathscr{A}_{1}$, onto the Banach algebra $\mathscr{A}_{2}$, and if the multiplication in $\mathscr{A}_{1}$ is regular, then so is the multiplication in $\mathscr{A}_{2}$.

\section{Proof. Civin and Yood [2], Corollary 6.4.}

Corollary 4.1. If $X$ is a Banach space with an unconditional basis $\left(e_{j}\right)$, and which is isomorphic to a uniformly convex space, then its space of compact operators is Arens regular.

Proof. By Proposition 2.1., $X$ can be renormed isomorphically to $X^{\prime}$ so that $\left(e_{j}\right)$ is an unconditionally monotone basis for $X^{\prime}$. Let $i$ be an isomorphic map from $X$ to $X^{\prime}$. Then the map $A \mapsto i^{-1} A i$, where $A \in \mathscr{C}^{\prime}$, is a continuous homomorphism from $\mathscr{C}^{\prime}$ onto $\mathscr{C}$.

THEOREM 4.2. Let $X$ be a Banach space with an unconditionally monotone, shrinking basis, and for which the matrices in $\mathscr{C}^{*}$ with a finite number of rows are norm dense. Then $B(X)=\left\{F \in \mathscr{C}^{* *}\right.$ : $F_{1}^{*} A$ and $A_{1}^{*} F$ are both in $\mathscr{C}$ for all $\left.A \in \mathscr{C}\right\}$. Furthermore, each of the Arens products coincides with operator multiplication on $B(X)$.

Proof. Let $F$ be in $\mathscr{C}^{* *}$. Let $D_{j}$ denote the elementary matrix $E_{j j}$. Call $D_{j 1}^{*} F$ the $j^{\text {th }}$ row of $F$. Note that $D_{j 1}^{*} F$ is concentrated on the $j^{\text {th }}$ row of matrices in $\mathscr{C}^{*}$. In fact,

$$
\left(D_{j 1}^{*} F\right) f=D_{j}\left(F_{1}^{*} f\right)=\left(F_{1}^{*} f\right) D_{j}=F\left(f_{1}^{*} D_{j}\right) .
$$

But the matrix for $f_{1}^{*} D_{j}$ is easily seen to be the matrix formed from $f$ by deleting all but the $j^{\text {th }}$ row. By Proposition 2.7., the $j^{\text {th }}$ row of $F$ can be identified with a functional in $X^{* * *}$.

Call $F_{1}^{*} D_{j}$ the $j^{\text {th }}$ column of $F$. It is concentrated on the $j^{\text {th }}$ column of matrices in $\mathscr{C}^{*}$, because $D_{j 1}^{*} f$ is the matrix formed by deleting all but the $j^{\text {th }}$ column of $f$. Then by Proposition 2.7. it can be identified with an element of $X^{* *}$.

We claim $F \in B(X) \Longleftrightarrow$ each of its rows is in $X^{*}$ and each of its columns is in $X$. Suppose $F \in \mathscr{C}^{* *}$ with each of its rows in $X^{*}$ and columns in $X$. Let $T$ be the actual matrix formed by writing down the columns of $F$ as elements in $X$ with respect to the basis $\left(e_{j}\right)$. Let $T_{n}$ be the first $n$ columns of $T$. It is a compact operator since each column is in $X$. Also by Proposition 2.6. 


$$
\left\|T_{n}\right\|=\left\|\Phi_{T_{n}}\right\| \leqq\|F\|
$$

where $\Phi$ is the isometry defined in Theorem 3.1. Hence, the $\left\{T_{n}\right\}$ define a single bounded operator on the dense linear manifold of finite linear combinations of $\left(e_{j}\right)$. This bounded operator has the same matrix as $T$.

Clearly $\Phi_{T}$ and $F$ agree on any elementary matrix in $\mathscr{C}^{*}$. Hence they agree on any matrix in $\mathscr{C}^{*}$ concentrated in a single row, since each row of $F$ is in $X^{*}$ and the $\left(e_{j}^{*}\right)$ form a basis for $X^{*}$. Then by the hypothesis that the matrices in $\mathscr{C}^{*}$ with a finite number of rows are dense, $\Phi_{T}=F$.

Conversely, if $F \in B(X)$ it is clear that its generalized rows and columns will be in $X^{*}$ and $X$ respectively.

Using this characterization of $B(X)$ as a subspace of $\mathscr{C}^{* *}$, it is clear that if $F \notin B(X)$, then for some $j$ either $D_{j 1}^{*} F$ or $F_{1}^{*} D_{j}$ lies outside $B(X)$ and hence outside $\mathscr{C}$. But $D_{j}$ is a compact operator.

To finish the proof we will show that on $B(X), m_{1}$ is equal to operator multiplication. The proof for $m_{2}$ is similar.

Clearly it is enough to show that $(S T) f_{j}=\left(S_{1}^{*} T\right) f_{j}$ for $f_{j}$ a matrix in $\mathscr{C}^{*}$ concentrated in the $j^{\text {th }}$ row and where $\|S\|=\|T\|=\left\|f_{j}\right\|=1$. Given $\varepsilon>0$, we can approximate the $j^{\text {th }}$ row of $S$ in norm to within $\varepsilon$ by deleting after the first $n$ terms for $n$ large enough.

Then

$$
\begin{aligned}
(S T) f_{j} & =\left(S_{j 1} T_{11}+S_{j 2} T_{21}+\cdots+S_{j n} T_{n 1}\right) f_{j 1} \\
& \vdots \\
& +\left(S_{j 1} T_{1 k}+S_{j 2} T_{2 k}+\cdots+S_{j n} T_{n k}\right) f_{j k} \\
& \vdots \\
& +(\text { error term }<\varepsilon) .
\end{aligned}
$$

We claim that $\left(T_{1}^{*} f_{j}\right)$ is concentrated in the $j^{\text {th }}$ row. In fact,

$$
\left(T_{1}^{*} f_{j}\right) E_{m k}=T\left(f_{j 1}^{*} E_{m k}\right)=0 \text { if } m \neq j,
$$

whereas $\left(T_{1}^{*} f_{j}\right) E_{j k}=\operatorname{dot}$ product of $k^{\text {th }}$ row of $T$ with $j^{\text {th }}$ row of $f_{j}$. Then,

$$
\begin{aligned}
S\left(T_{1}^{*} f_{j}\right) & =\left(T_{11} f_{j 1}+T_{12} f_{j 2}+\cdots+\right) S_{j 1} \\
& \vdots \\
& +\left(T_{n 1} f_{j 1}+T_{n 2} f_{j 2}+\cdots+\right) S_{j n} \\
& +(\text { error term }<\varepsilon) .
\end{aligned}
$$

Hence $\left|(S T) f_{j}-\left(S_{1}^{*} T\right) f_{j}\right|<2 \varepsilon$, since for a finite collection of convergent series 


$$
\sum_{k=1}^{\infty}\left(a_{k}^{1}+\cdots+a_{k}^{n}\right)=\sum_{k=1}^{\infty} a_{k}^{1}+\cdots+\sum_{k=1}^{\infty} a_{k}^{n}
$$

DEFinition 4.6. A shrinking basis $\left(e_{j}\right)$ for a Banach space is called boundedly growing if there exists an $\varepsilon>0$ and an integer $n$, such that $x_{1}+\cdots+x_{n}<n-\varepsilon$ whenever the $x_{i}^{\prime}$ s have norm 1 and are linear combinations of distinct basic vectors. For example the canonical bases for $c_{0}$ or $l^{p}, p>1$ are boundedly growing. Finite direct sums of boundedly growing Banach spaces are boundedly growing. Also $l^{p}\left(X_{i}\right)$ for $p>1$ is boundedly growing if the $X_{i}$ have a common $n$ and $\varepsilon$.

COROLlary 4.2. If a Banach space $X$ has an unconditionally monotone, boundedly growing basis then $B(X)$ is the largest subset in $\mathscr{C}^{* *}$ in which $\mathscr{C}$ is a two sided ideal.

Proof. In proving Proposition 3.3. we showed that if the finite matrices are not dense in $\mathscr{C}^{*}$ then the basis is not boundedly growing. Similarly, if the matrices with a finite number of rows are not dense in $\mathscr{C}^{*}$, then the basis is not boundedly growing.

\section{BIBLIOGRAPHY}

1. R. F. Arens, The adjoint of a bilinear operation, Proc. Amer. Math. Soc. 2 (1951), 839-848.

2. P. Civin and B. Yood, The second conjugate of a Banach algebra as an algebra, Pacific J. Math. 11 (1961), 847-870.

3. J. Clarkson, Uniformly convex spaces, Trans. Amer. Math. Soc. 40 (1936), 396-414.

4. M. M. Day, Normed linear spaces, Academic Press, New York, 1962.

5. J. Hennefeld, A note on the Arens product, Pacific J. Math. 26 (1968), 115-119.

6. I. Maddaus, On completely continuous linear transformations, Bull. Amer. Math. Soc. (1938), 279-282.

7. A. Pelczynski, Projections in certain Banach spaces, Studia Math. 19 (1960), 209228.

8. C. E. Rickart, General theory of Banach algebras, Van Nostrand, Princeton, 1960.

9. R. Schatten, Norm ideals of completely continuous operators, Springer-Verlag, Berlin (1960).

10. A. Wilansky, Functional analysis, Blaisdell, New York, (1964).

Received March 4, 1968, and in revised Form July 30, 1968. The material in this paper is part of the author's doctoral dissertation written at Columbia University under the guidance of Prof. E. R. Lorch, and was supported by NSF grant GP-9003. 



\section{PACIFIC JOURNAL OF MATHEMATICS}

\section{EDITORS}

H. ROYDEN

Stanford University

Stanford, California

\author{
R. R. Phelps \\ University of Washington \\ Seattle, Washington 98105
}

J. DugundJI

Department of Mathematics

University of Southern California

Los Angeles, California 90007

RICHARD ARENS

University of California

Los Angeles, California 90024

\section{ASSOCIATE EDITORS}

E. F. BECKenbaCH

B. H. NEUMANN

F. WOLF

K. YoSHIDA

\section{SUPPORTING INSTITUTIONS}

\author{
UNIVERSITY OF BRITISH COLUMBIA \\ CALIFORNIA INSTITUTE OF TECHNOLOGY \\ UNIVERSITY OF CALIFORNIA \\ MONTANA STATE UNIVERSITY \\ UNIVERSITY OF NEVADA \\ NEW MEXICO STATE UNIVERSITY \\ OREGON STATE UNIVERSITY \\ UNIVERSITY OF OREGON \\ OSAKA UNIVERSITY \\ UNIVERSITY OF SOUTHERN CALIFORNIA
}

\author{
STANFORD UNIVERSITY \\ UNIVERSITY OF TOKYO \\ UNIVERSITY OF UTAH \\ WASHINGTON STATE UNIVERSITY \\ UNIVERSITY OF WASHINGTON \\ $\stackrel{*}{*} \stackrel{*}{*} \stackrel{*}{*}$ AMERICAN MATHEMATICAL SOCIETY \\ CHEVRON RESEARCH CORPORATION \\ TRW SYSTEMS \\ NAVAL WEAPONS CENTER
}

The Supporting Institutions listed above contribute to the cost of publication of this Journal, but they are not owners or publishers and have no responsibility for its content or policies.

Mathematical papers intended for publication in the Pacific Journal of Mathematics should be in typed form or offset-reproduced, double spaced with large margins. Underline Greek letters in red, German in green, and script in blue. The first paragraph or two must be capable of being used separately as a synopsis of the entire paper. It should not contain references to the bibliography. Manuscripts, in duplicate if possible, may be sent to any one of the four editors. Please classify according to the scheme of Math. Rev. 36, 1539-1546. All other communications to the editors should be addressed to the managing editor, Richard Arens, University of California, Los Angeles, California, 90024.

50 reprints are provided free for each article; additional copies may be obtained at cost in multiples of 50 .

The Pacific Journal of Mathematics is published monthly. Effective with Volume 16 the price per volume (3 numbers) is $\$ 8.00$; single issues, $\$ 3.00$. Special price for current issues to individual faculty members of supporting institutions and to individual members of the American Mathematical Society: $\$ 4.00$ per volume; single issues $\$ 1.50$. Back numbers are available.

Subscriptions, orders for back numbers, and changes of address should be sent to Pacific Journal of Mathematics, 103 Highland Boulevard, Berkeley, California, 94708.

PUBLISHED BY PACIFIC JOURNAL OF MATHEMATICS, A NON-PROFIT CORPORATION

Printed at Kokusai Bunken Insatsusha (International Academic Printing Co., Ltd.), 7-17, Fujimi 2-chome, Chiyoda-ku, Tokyo, Japan. 


\section{Pacific Journal of Mathematics \\ Vol. 29, No. $3 \quad$ July, 1969}

Herbert James Alexander, Extending bounded holomorphic functions from certain subvarieties of a polydisc ...................... 485

Edward T. Cline, On an embedding property of generalized Carter

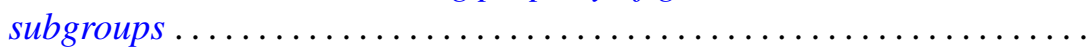

Roger Cuppens, On the decomposition of infinitely divisible characteristic functions with continuous Poisson spectrum. II ...............

William Richard Emerson, Translation kernels on discrete Abelian

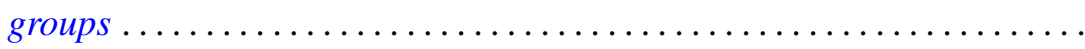

Robert William Gilmer, Jr., Power series rings over a Krull domain ....... 543

Julien O. Hennefeld, The Arens products and an imbedding theorem ...... 551

James Secord Howland, Embedded eigenvalues and virtual poles ........ 565

Bruce Ansgar Jensen, Infinite semigroups whose non-trivial homomorphs are all isomorphic .............................. 583

Michael Joseph Kascic, Jr., Polynomials in linear relations. II .......... 593

J. Gopala Krishna, Maximum term of a power series in one and several

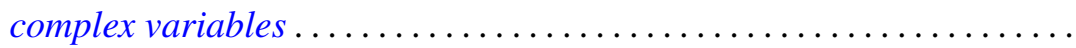

Renu Chakravarti Laskar, Eigenvalues of the adjacency matrix of cubic lattice graphs ...................................

Thomas Anthony Mc Cullough, Rational approximation on certain plane

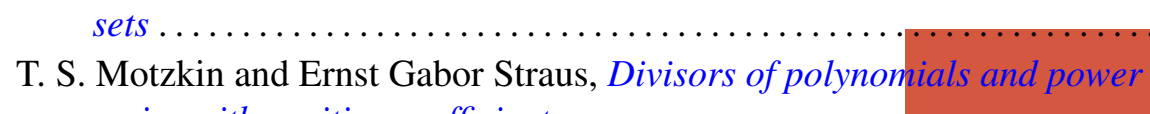
series with positive coefficients .

Graciano de Oliveira, Matrices with prescribed characteristic polynomial and a prescribed submatrix.

Graciano de Oliveira, Matrices with prescribed characteristic polynomial and a prescribed submatrix. II .

Donald Steven Passman, Exceptional 3/2-transitive permutation groups .................................

Grigorios Tsagas, A special deformation of the metric with no negative sectional curvature of a Riemannian space............

Joseph Zaks, Trivially extending decompositions of $E^{n}$ 